\title{
Zur Hadesfahrt Christi.
}

\author{
Von + Wilhelm Bousset in Gießen ${ }^{2}$.
}

In einem Anhang des ersten Kapitels meines Kyrios Christos hatte ich auf wenigen Seiten das Problem der "Hadesfahrte behandelt. Das Ziel meiner Ausführungen war der Nachweis, daß hier ein orientalischer Mythos frühzeitig in den christlichen Glauben eingedrungen sei. Um das deutlicher zu machen, schied ich zwischen zwei Strömen der Überlieferung. Ich charakterisierte die bei den Kirchenvätern herrschende Vorstellung von der Hadespredigt als ein bereits > von des Gedankens Blässe angekränkeltes Theologumenon «, das vorwiegend der theologischen Literatur angehöre und unterschied davon eine mehr unliterarische volkstümliche Überlieferung, in welcher der. Mythos yon einem Erlöserheros, der zum Kampf mit den Dämonen in die Tiefen der Unterwelt hinabstieg, noch deutlicher sich abspiegle. Ich glaubte hier - trotz entgegenstehender literarischer Überlieferung - das Ursprüngliche erblicken zu sollen, die volkstümliche Aneignung des ursprünglichen Mythos, und betonte, wie die Kraft und Originalität des christlichen "Geistes * darin bestanden habe, daß er aus dem Mythos die Idee von einer alle Menschengeschlechter umfassenden Ekklesia, dem Universalismus des Heils gestaltet habe.

Gegen diese Auffassung und Behandlung der Frage hat Carl Schmidt in einem Exkurs (II) seiner Ausgabe der ${ }$ Gespräche Jesu mit seinen Jüngern nach der Auferstehung \& 2 lebhaften Einspruch

${ }^{1}$ [Als mir der Verfasser den nachstehenden Aufsatz zusagte, stand er in voller Lebens- und Arbeitskraft vor mir, und nicht minder, als er die letzte Frage in diesem Leben an mich richtete, eine Frage nach der.Drucklegung dieser Arbeit, die wohl das letzte ist, was er für den Druck vorbereitet hat. Nur wenige Tage später, am 15. März; nahm ein plötzlicher Tod dem Unermüdlichen die Feder aus der Hand. Ein allen unerwarteter Schlag ließ dies treue und starke Herz stille stehen. Die Zeitschrift betrauert in dem Entschlafenen nicht nur einen ihrer ältesten Mitarbeiter, der sich mit einer tief grabenden Abhandlung über die Testamente der 12 Patriarchen schon am 1. Jahrgang beteiligt hat, sondern auch den anregenden Forscher, der dic wissenschaftliche Arbeit am NT wie kaum ein anderer befruchtet hat. Denn jedes Problem, das er in energischer Arbeit bewältigte, offenbarte ihm wieder eine Fülle neuer Probleme. Was er in reicher Lebensarbeit der Wissenschaft geschenkt hat, wird fortwirken und noch fruchthar sein, auch wenn sich Methode und Ziele der Forschung schon längst wieder geändert haben.

E. P.]

2 Texte und Untersuchungen III. Folge. Bd. XIII. 
erhoben. Er versucht, mir in diesem Exkurs von über I20 Seiten (S. 452-576) nachzuweisen, daß ich die Dinge vollkommen auf den Kopf gestellt habe, daß es sich hier um Herübernahme eines fremden Mythos überhaupt nicht handle, daß der Gedanke der Heilspredigt Jesu nicht ein Theologumenon darstelle, sondern einfachen und weitverbreiteten Gemeindeglauben, nicht smüßiges Gebilde der Phantasie und schattenhaftes Mythologumenon* sondern tiefgewurzelte Stimmung, welche die Menschen mit ihrer Religion verbinden «, - daß jene lebhafteren volkstümlichen Vorstellungen spätere Singularitäten, dichterische Einfälle einer freischwebenden Phantasie seien, an denen der Historiker, der auf die großen Linien achte, getrost vorübergehen könne.

Ich habe mich von dem Recht der Beweisführung Schmidts nicht überzeugen können und sehe mich genötigt, die Auseinandersetzung. auf der ganzen Linie aufzunehmen. In dem weitaus größeren Teil seines 2 Exkurs̀es ( $\mathrm{S}$. 443-546) sucht Schmidt durch ein ausführliches Verhör der Zeugen den Nachweis zu erbringen, daß gerade der Gedanke der befreienden Hadespredigt Christi urchristlichen und zwar genuin urchristlichen Gemeindeglauben darstelle. Ich gestehe, daß es mir schwer wird, aus der Unmenge der hier angestellten Erörterungen unter Beiseitelassung des Ballastes, mit dem mein Gegner sein Schiffchen befrachtet hat, das eigentliche Wesentliche seiner Beweisführung herauszugreifen. - Oft scheint es fast so, als genüge ihm die Feststellung einer gewissen Stabilität und Gleichförmigkeit, mit der von seinen Zeugen die Gedanken über die Hadesfahrt Christi vorgetragen werden, um auf Grund dessen seine These des Gemeindeglaubens zu erheben. Besonders deutlich ist das bei seiner Zusammenfassung S. $546 \mathrm{f}$.: $\gg$ Durch diese Art der Behandlung sollte deutlich vor die Augen treten, was christlicher Glaube, was dogmatisch gelehrtes Beiwerk der Theologen ist'.* Ụnd nun nennt er als Theologumena: die Predigt Christi an die ungehorsamen Geister I Pt 3, I8, die Predigt der Apostel in der Unterwelt im Hirten des Hermas, die Predigt Christi an die gerechten Seelen der Heiden bei Clemens usw. Das sei Rankenwerk. Klar jedoch hebe sich oder christliche Glaube ab «, und zu ihm gehöre das Eingehen der abgeschiedenen Seele Jesu in den Hades, dessen heilsgeschichtliche Tätigkeit während des dreitägigen Aufenthalts, d. h. die Verkündigung des Heils an die alttestamentlichen Gerechten, ihre Aufnahme in die Gemeinde durch die Taufe (!), ihre Befreiung aus der Unterwelt und Aufstieg in die

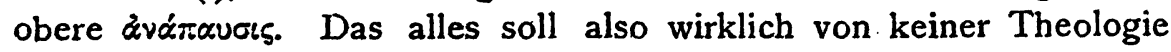
angekränkelter Gemeindeglaube sein: "Stimmungen, welche die 
Mendchen mit ihrer Religion verbinden. « Und aus welchem Grunde? Weil es überall, weil es immer gesagt wird! So kann man in der Tat manches Theologumenon zum Range des Gemeindeglaubens erheben, denn auch Theologumena können bekanntlich recht stabil und weit verbreitet sein. Sie können auch in populären und halbpopulären Schriften ihren Nachklang und Widerhall finden, so daß es kaum ein Beweis für Schmidt sein dürfte, daß unter den Zeugen für die Hadespredigt sich auch z. B. das Petrusevangelium, die von ihm behandelte, übrigens reichlich theologische, neue apokryphe Schrift und die Sibylle neben so manchen spezifisch theologischen Zeugen findet. Auch kann mich Schmidts Hinweis auf die Gnostiker, vor allem auf Marcion und dessen boshafte und polemische Umkehr der Tendenz der Hadespredigt (Irenaeus I 27,3) nicht beirren. Schmidt argumentiert zwar (S. 483): „Wäre der Descensus behufs Erlösung der alttestamentlichen Frommen nur ein von des Gedankens Blässe angekränkeltes Theologumenon gewesen, hätte schwerlich Marcion seine Spezialansichten zum Ausdruck gebracht. Jetzt aber sah er sich genötigt, dem. Gemeindéglaube n Rechnung zu tragen.* Ich kann hier kein anderes Zugeständnis machen, als daß Marcions Polemik eine gewisse Verbreitung und Popularisierung der üblichen Auffassung von der Hadesfahrt Jesu voraussetzt; aber damit ist über Herkunft und Charakter dieser noch gar nichts ausgesagt. Und dasselbe habe ich wiederum zu sagen, wenn Schmidt S. 498 Celsus mit seiner Polemik als Kronzeugen für seine These anführt. Hat Celsus etwa niemals Theologumena der von ihm bekämpften Religion bespöttelt?!

Schmidts hundertseitige Gelehrsamkeit vernag mir das einfache Geschmacksurteil nicht zu verschütten. Man sehe sich nur den oben zitierten Satz (auf S. 546 f.) noch einmal an. Was für ein merkwürdiger Gemeindeglauben ist das doch, $z u$ dessen unmittelbarem Inhalt etwa die Taufe der alttestamentlichen Gerechten durch den zum Hades gefahrenen Christus gehört haben soll. Was hat schon an und für sich die Hadesfahrt mit lebendigem unmittelbarem Gemeindeglauben zu tun, zumal wenn Schmidts Charakterisierung richtig wäre: ^Nach urchristlicher Überlieferung ist nicht ein Heros oder Königssohn, nicht ein Gott zu den Toten herabgestiegen .... sondern ein Mensch, der den Tod am Kreuze erlitten hat und nun wie jeder Sterblich.e das Schicksal der Abgeschiedenen teilt. Und was hat das Geschick der alttestamentlichen Gerechten vor allem mit dem unmittelbaren Glauben der heidenchristlichen Kirche $z u$ schaffen? Ja wenn wir den Gedanken bei Paulus fänden, innerhalb der Stim- 
mungswelt von Röm 9-II, oder in jụdenchristlichen Quellen! Doch es mag zugestanden werden: ein indirektes Interesse mag christlichem Gemeindeglauben am Geschick der alttestamentlichen Frommen zugebilligt werden. Die Auffassung von einer Heilsgeschichte, welche ihren Ausgangspunkt bei den alttestamentlichen Patriarchen nimmt. und für die das Geschick dieser Heroen auch der christlichen Religion nicht gleichgültig sein kann, die Idee einer Kirche, einer Gemeinschatt, welche die Toten und die Lebendigen umfaßt, sind Gedanken, die dem unmittelbaren Glauben nicht fernliegen. Aber immerhin es sind bereits reflektierte Gedanken. Und nun gar die Art, wie das Dilemma durch die Annahme einer Predigt Christi im Hades gelöst wird, trägt doch den Charakter einer sehr künstlichen Reflexion, einer Reflexion, die dann als recht überflüssig erscheinen könnte, ẇènn "man sich erinnert, daß z. B. der paulinische Gedanke einer Leitung des alttestamentlichen Gottesvolkes durch den präexistenten Christus und die johanneische Behauptung von der Freude Abrahams am Tage des Messias jenes Prablem zu lösen imstande waren. So muß ich, trotz Schmidt, bei meiner.Beurteilung der Vorstellung von der Hadespredigt Christi als eines Theologumenons beharren.

Wichtiger ist freilich die zweite Fragestellung, die Schmidt mit der ersten verbindet. Mag nun die Predigt Christi im Hades ein Theologumenon oder ein wertvoller Bestand des Gemeindeglaubens sein: darauf kommt es uns in unserem Streit vor allem an, ob diese Phantasie aus genuinen urchristlichen (jüdischen) Prämissen begreifbar werde oder nicht. Und mein Kritiker hat wiederum eine Fülle von Gelehrsamkeit gegen mich aufgeboten, um diese Frage in ersterem Sinne zu beantworten. Er fordert (S. 454) von einem echten Religionshistoriker, daß er, um dieser Frage gerecht zu werden, zunächst sich die Aufgabe stelle, die Jenseitsvorstellungen des zeitgenössischen Judentums (und des Urchristentums) ans Licht zu stellen. Er unterzieht sich deshalb von neuem (S. 455-464) dieser Aufgabe, um einen sichern. Ausgangspunkt für seine Auffassung zu gewinnen. Ich hätte gegen seine Darstellung nun allerlei einzuwenden.

Ich möchte behaupten - und meine Beschäftigung mit dem Judentum gibt mir wohl das Recht zu einer Behauptung ohne Beweis, - daß er die Jenseitsvorstellungen des palästinensischen Judentums viel zu einseitig, systematisch dargestellt hat. Aber ich will das Bild, das er gezeichnet hat, stehen lassen. Ich will ihm sogar gern zugestehen, daß die Anschauung, daß Jesus zwischen Tod und Auferstehung eine kurze Frist - auf die drei Tage soll es nicht an- 
kommen - in der Ruhe des Hades zugebracht habe, wie jede Menschenseele vor ihrer Erweckung, durchaus jüdischen Prämissen entspreche. Schwieriger ist es schon, von hier aus den Gedanken der Hadespredig t abzuleiten. Schwieriger gerade dann, wenn mein Kritiker selbst so stark betont, daß nach urchristlicher Auffassung Jesus in den Hades gegangen sei wie ein Mensch, der den Tod erlitten und nun wie jeder Sterbliche das Schicksal der Abgeschiedenen teilt. Dazu will nun schon die Aktivität, die Christus mit der Hadespredigt zeigt; nicht recht passen. Aber Schmidt kann mir hier freilich mit dem Hinweis auf jenes urchristliche Interesse am Geschick der alttestamentlichen Frommen antworten. Beides zusammen, die urchristliche Auffassung vom Weilen Jesu im Hades und dieses Interesse habe auf geradem Wege zu jener Kombination geführt: Predigt Jesu im Hades. - Aber e in e s bleibt nun doch übrig, was gerade gar nicht in das so stark betonte Milieu des spätjüdisch-urchristlichen Gemeindeglaubens hineinpaßt, das ist der Gedanke der unmittelbaren Herausführung der durch die Predigt begnadeten Frommen aus dem Hades! Das steht gerade der jüdischen Durchschnittseschatologie, wie Schmidt sie selbst gezeichnet hat; 'genau entgegen. Denn diese gipfelt in dem Gedanken der leiblichen Auferstehung hier auf Erden, kennt bis dahin nur einen Schlummer- oder Zwischenzustand der Seelen im Hades, kennt den Gedanken einer himmlischen àvájavoıs jener und damit den einer vorzeitigen Entrückung der Seelen in diese überhaupt nicht. Und wenn ich auch gegen Schmidt oben geltend gemacht habe, daß selbst im palästinensischen Judentum die Dinge so einfach nicht liegen, so würde ich doch auch meinerseits betonen, daß überall, wo sich in der jüdischen Eschatologie Gedanken zeigen, die das Dogma von der leibhaftigen Auferstehung hier auf Erden und die damit im Zusammenhang stehende Idee von dem status intermedius im Hades durchbrechen, fremdartige Einflüsse vorliegen.

Auch den Einwand glaube ich von Schmidt nicht. erwarten $z u$ sollen, daß der Gedanke der Herausführung der Seelen aus dem Hades nicht zum ursprünglichen Bestand der in Frage kommenden Vorstellungen gehöre und etwa erst später willkürlich hinzugefügt sei. Er schwankt hier zwar das eine oder andre Mal (z. B. S. 490), aber er erklärt doch in seinem abschließenden Urteil gerade auch die Herausführung der Seelen aus dem Hades für einen festen Bestand des urchristlichen Glaubens (S. 546f.). Und nach den vorliegenden Quellen kann auch kaum anders geurteilt werden. Denn in den weitaus meisten und wichtigsten Zeugnissen - von dem ältesten, das uns zur Verfügung steht, dem des Ignatius an - ist in der Tat immer gerade 
von der Herausführung die Rede. Die Ausführungen des Hirten des Hermas ', das von Schmidt behandelte Apokryphon, der Häretiker Marcion, die Excerpta ex Theodoto, die Pistis Sophia, Hippolyt, die ägyptische Kirchenordnung wenigstens in ihrer äthiopischen Überlieferung, die Acta Thaddaei treten dem ältesten Zeugnis des Ignatius zur Seite. Wenn bei einigen Kirchenvätern diese Phantasie unterdrückt ist, so hat Schmidt selbst darauf hingewiesen, daß hier dogmatische Bedenken vom Standpunkt der spätjüdisch-altchristlichen Eschatologie mitsprechen.

Merkwürdig ist es nun, daß Schmidt das alles gesehen, und sich doch der Tragweite dieser Beobachtungen vollkommen verschlossen hat! Denn an ihm scheitert ja nun eigentlich seine Behauptung, daß die Idee der Hadesfahrt Jesu, wie sie nach ihm im Gemeindeglauben vörliegt, vollkommen aus jüdisch-urchristlichen Prämissen ableitbar und verständlich sei. Es hat sich hier ein Fremdkörper gezeigt, den man doch nicht einfach als gegeben hinnehmen darf, der uns zu weiterer Nachforschung zwingt.

Dieser Neigung Schmidts, an unbequemen Tatsachen mit geschlossenen Augen vorüberzugehen, entspricht nun vor allem seine Behandlung desjenigen Zeugnisses, das doch vor allem, wenn nicht als das älteste, so doch als eines der ältesten, zum mindesten als das einzige neutestamentliche, das die Hadespredigt Christi be sti mm t ausspricht, eine besondere Aufmerksamkeit und Behandlung verdient. Ich meine die Stelle I Pt 3, 19, $(4,16)^{2}$. Es ist wenig genug, was uns Schmidt darüber zu sagen weiß. Er erklärt, die exegetischen Bemühungen hätten hier eine befriedigende Erklärung nicht zutage gefördert. Der Grund liege darin, daß der Verfasser die ihm bekannte Idee der Hadesfahrt Christi für seine besondere Paränese benutzt und daher den genuinen Gedanken alteriert habe. Daher sei es ein vergebliches Unterfangen, den Gedanken des Verfassers klar zu erfassen, insbesondere dürfe man die Stelle I Pt 3, 19 nicht zum Ausgangspunkt bei einer Darstellung der Descensus-Idee machen (S. 464).

An diesem Punkt haben wir also, um weiterzukommen, die Einzeluntersuchung noch einmal aufzunehmen. Ich stelle den Wortlaut der

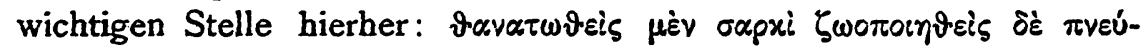

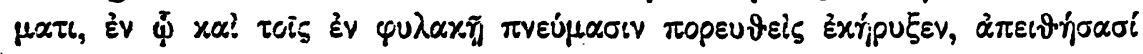

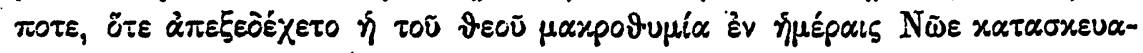
Irenaeus.

1 Nicht sicher ist das Urteil über das gefälschte Jeremiaszitat bei Justin und

2 Hier hat selbst Harnack sein Bedenken gegen die Beweisführung Schmidts ausgesprochen. Vgl, dessen Besprechung Theol. Lit.-Zeit. 1919 Nr. 22. 


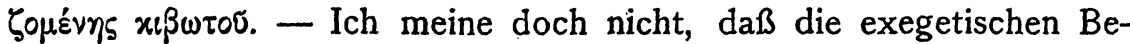
mühungen um diese Stelle so erfolglos gewesen seien, wie Schmidt es dekretiert. Vor allem scheinen mir zunächst diejenigen Forscher ${ }^{1}$ recht $z u$ haben, die behaupten, daß sich die $\pi v \varepsilon u ́ \mu \alpha \tau \alpha$ żv $\varphi v \lambda \alpha \times \tilde{n}$ nicht auf verstorbene Menschenseelen, und die Predigt an die $\pi \nu \varepsilon u ́ \mu \alpha \tau$ nicht auf die sonst geläufige Vorstellung von der Hadespredigt Christi be-

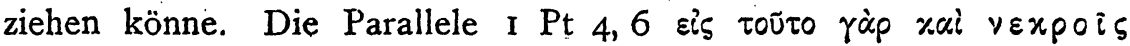

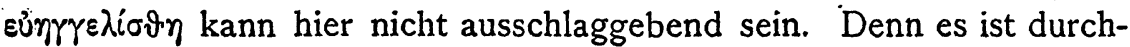
aus nicht gesagt, daß es sich I Pt 3, I9 und 4, 6 um genau denselben Vorgang innerhalb der $Z$ wischenzeil zwischen Tod und Auferstehung Jesu handeln müsse. Die Bezeichnung der Verstorbenen als $\pi v \varepsilon u ́-$ $\mu \alpha \tau \alpha$ (nicht vexpol oder $\psi \cup \times \alpha i$ ) würde zum mindesten eine gewisse Singularität innerhalb des vom Judentum her bestimmten Urchristentums bedeuten. Auch der Ausdruck Geister »im Gefängnis w wäre innerhalb jüdisch bestimmter Eschatologie im höchsten Grade merkwürdig bei verstorbenen Seelen, auch dann, wenn es sich um Seelen der Bösen handelt. Daher wird man die $\pi v \varepsilon u ́ \mu \alpha \tau \alpha$ हैं $\varphi$ auf die ungehorsamen Menschengeschlechter zu Zeiten Noahs deuten dürfen, sondern vielmehr auf die aufrührerischen dämonischen Mächte, die nach Genesis 6 in den Tagen vor der Sintlut ihr Wesen trieben, und von denen die Henochbücher und Jubiläen ausführlich zu berichten wissen. Eine direkte und entscheidende Bestätigung bietet uns $2 \mathrm{Ft} \mathrm{2,6}$, wo wiederum unmittelbar vor Noah die sündigen Engel erwähnt werden, die Gott in Fesseln der Finsternis (finsteren Höhlen) dem Tartarus zur Aufbewahrung für das Gericht überwiesen habe, und

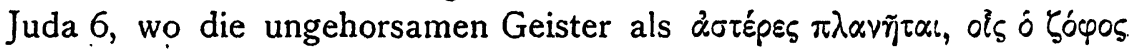

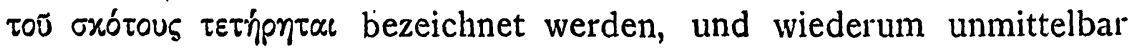
daneben von der Gerichtspredigt Henochs an diese die Rede ist. So werden wir in der Tat mit diesem ganzen Gedankenkomplex in erster Linie an die mythologischen Phantasien des ersten Abschnittes des Buches Henoch gewiesen. Man würde aber andrerseits fehlgehen, wollte man mit manchen Forschern annehmen, es handle sich hier schließlich gar nicht direkt um eine Hadespredigt Christi, d. h. um ein Ereignis zwischen Tod und Auferstehung Christi, sondern um die Bußpredigt, die der präexistente Christus in der Figur des Henoch den gefallenen Engeln gehalten habe. Dem widerspricht auf das be-

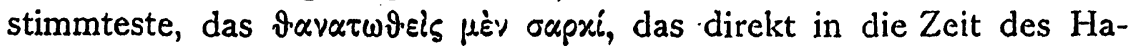

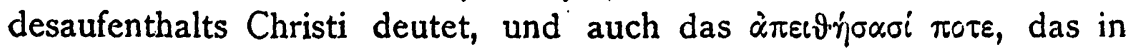
eine ferne Vergangenheit weist. Was hier vorliegt, ist nicht die Identifikation des präexistenten Christus mit der Figur Henochs, son-

1 Vgl. hier vor allem Gunkels Ausführungen in den sSchriften des NT\& zur Stelle. 
dern die Übertragung des Henochmythos auf den Aufenthalt Jesu in der Unterwelt.

Dann aber ist es schon jetzt klar, daß es sich in I Pt 3, I9 um die Übertragung eines fremdartigen und noch nachweisbaren Mythos auf die Person Jesu und sein Geschick im Hades handelt; klar auch der geheimnisvolle und nur kurz andeutende Ton, in dem auf diesen vom Verfasser des Briefes angespielt wird. Ebenso deutlich aber ist es weiterhin, daß diese Erzählung von den revolutionierenden Engeln, ihrer Einkerkerung und der Gerichtspredigt Henochs nicht der freien Phantasie einer auf Genesis 6 beruhenden jüdischen Haggada entstammt. Es sind die kräftigen Züge eines orientalischen Wandermythos, die uns in der Erzählung anblicken. Und wir dürfen weiter vermuten, daß es sich in diesem orientalischen Mythos nicht um gefallene Engel; sondern um revoltierende böse Göttermächte oder Dämonen handelt, und nicht un einen sagenhaften Patriarchen der Urzeit, sondern um einen Gott-Heros oder Gott, der zum Hades hinabfährt. Weiß doch auch das Henochbuch von der Erhöhung Henochs zum Menschensohn, einer gottähñlichen, halbgöttlichen $\mathrm{Ge}$ stalt, $\mathrm{zu}$ berichten.

Und wir sind denn, wie ich glaube, nunmehr in der glücklichen Lage, hier nicht blo13 bei Vermutungen stehen bleiben zu können. Seitdem ich meinen Abschnitt im. Kyrios schrieb, hat sich unser Material um eine wichtige neue Entdeckung bereichert. In den Proceedings of the Society of biblical Archaeology (Bd. 30, 1908, S. $59 \mathrm{ff}$.) veröffentlicht und bespricht Pinches einen neubabylonischen Text, den er sthe legend of Merodach « betitelt. Nach einer nicht ganz verständlichen Zeile am Anfang, von einem göttlichen Wesen, das nach dem Zusammenhang kaum ein anderes als Marduk sein kann, heißt es:

Er steigt hernieder in das Gefängnis,

Er erhebt sich (?) und erreicht das Gefängnis,

Er öffnet die Tore des Gefängnisses, er tröstet sie,

Da blickte er hin auf sie, sie alle; er freut sich.

Da schauten die gefangenen Götter auf ihn,

Freundlich sie alle - betrachteten sie ihn.

Leider wird der Text in seinem weiteren Verlauf schwer verständlich. Die scheinbar freundliche Szene wandelt sich. Es ist weiterhin die Rede von dem Eingreifen des Gottes Nergal. Der (oder Marduk selbst ?) verkündet, wie es scheint, eine Drohung (Marduks) gegenüber En-me-b̆ara, daß dessen $s$ i e b e n (I) Kinder alsbald 
vernichtet werden sollen. Darauf bricht En-me-šara in lautes Wehklagen aus ${ }^{1}$.

Dieser neuentdeckte Text wird nun aller Wahrscheinlichkeit mit einem Passsus der siebenten Tafel (Z. 27) des babylonischen Schöpfungsberichts zusammenzubringen sein (vgl. Pinches p. 59). Hier heißt es von Marduk, der unmittelbar vorher das Prädikat erhält, daß er aus dem Tode Leben schafft:

Er, der den gefangen gesetzten Göttern Gnade erwies,

Das Joch fortnahm, das auf den Göttern, die seine Feinde waren, lag,

Sie zu erlösen.

Wer hier die gefangenen Götter sind, wissen wir. Es können keine andern sein, als die Helfer der Tiâmat, von deren Gefangensetzung Tafel IV I10 ff, 127, berichtet. Und so hätten wir hier denn einen Mythos von der Befreiung der gefangenen Götter durch Marduk, und wenn die obige Kombination richtig ist, auch die gnädige Verkündigung der Befreiung durch Marduk im `Gefängnis «! - Im Henochbuch wird nun freilich den gefallenen Engeln im Gefängnis nicht Gnade und Befreiung angekündigt, sondern erbarmungsloses Gericht "Sage ihnen also: ihr werdet keinen Frieden haben* (164). Und so schwankt man bekanntlich auch, ob das ex ýpu $\xi_{\varepsilon v}$ von I Pt 3 , 19 Heilsoder Gerichts-Predigt bedeuten solle. Aber wir sahen ja auch bereits, wie in -der sLegend of Merodach«, jene freundliche Szene, die eine Begnadigung der gefangenen Götter anzudeuten schien, fast unvermittelt in eine furchtbare Gerichtsdrohung für En-me-šara und seine sieben Kinder übergeht. Es scheinen hier verschiedene Traditionen nebeneinander herzulaufen, von denen bald die eine, bald die andere nachgewirkt haben mag.

Für den Vergleich 2 wischen dem babylonischen Mythos und dem Henochbuch mag im Vorbeigehen noch auf das 19. Kapitel des Henochbuches verwiesen werden, wo berichtet wird, daß Henoch an einem grausigen Ort si eb e n Sterne wie große brennende Berge geschaut habe. "Ein Gefängnis ist dies für die Sterne .... die Sterne, die über dem Feuer dahinrollen, das sind die, welche beim Beginn ihres Aufgangs den Befehl Gottes übertreten haben. Da wurde er zornig und band sie 10000 Jahre* (vgl. den parallelen Bericht c. 21 ). Es verlockt sehr, den Beziehungen, die hier vorliegen, weiter nachzugehen. Daß auch die sieben Söhne des En-me-šara in dem babylonischen Text, denen die Gerichtsdrohung zuteil wird, Sterne seien,

1 Vgl. auch zu dieser Inhaltsangabe: H. Zimmern, Zum babylonischen Neujahrsfest. (Ber. i. d. Verhandl. d. sächs. Ges. d. Wissensch. Bd. 70. H. 5 1918 S. 49 f.) 
ist eine bereits mehrfach ausgesprochene Vermutung ${ }^{1}$. Außerdem wäre hier zu erinnern an den von mir in den Hauptproblemen der Gnosis S. $47 \mathrm{ff}$. ausführlich besprochenen persischen Mythos von den beim Ansturm Ahrimans auf den Himmel gefangenen Planetengöttern. Endlich wäre auch auf die Serie von Jōšamin-Lieder im Anfang des Johannesbuches der Mandäer (Lidzbarskis Uebersetzung S. $16 \mathrm{ff}$.) zu verweisen. Aber ich muß mich bei der Knappheit des. Raumes, der mir zur Verfügung steht, beschränken; glaube aber so viel deutlich gemacht zu haben, daß von I Pt 3, I9 und den Parallelen im Henochbuch die Linien deutlich und erkennbar in das Milieu eines orientalischen Mythos hinüberlaufen. Es wäre dann aber weiter reinste Willkür, diese älteste und markanteste Stelle, die von der Hadesfahrt Christi handelt, gegenüber der späteren Entwickelung der „Lehre، von der Hadesfahrt zu isolieren und zu verkennen, daß hier ein großer Zusammenhang vorliegt, an dessen Ende das christianisierte und theologisierte Dogma von der Hadespredigt Christi steht. Damit hat sich allerdings auch meine Beweisführung etwas verschoben, so freilich, daß meine religionsgeschichtliche Gesamtposition nur gewonnen hat. Gerade zu der Lehre von der Hadespredigt, die ich nur als theologische Abstraktion volkstümlicherer Vorstellungen auffaßte, finden sich direkte religionsgeschichtliche Parallelen. Ich hätte den Umweg meiner Beweisführung gar nicht nötig gehabt. Es gab einen Mythos von der Predigt (Heilspredigt resp. Gerichtsverkündigung) eines Gottes (Heros) an die Geister im Gefängnisse, als dessen direkter Zeuge in der christlichen Literatur I Pt 3, 19, in der jüdischen das Henochbuch anzusprechen ist:

Ich halte aber auch die Behauptung aufrecht, daß es daneben einen noch wilderen, leidenschaftlicheren und volkstümlicheren Mythos gegeben hat, der von dem Kampf und Sieg eines Gottes (Heros) gegenüber den Dämonen der Tiefe handelte und der ebenfalls seine breiten Spuren in dem volkstümlichen $Z$ weig der christlichen Literatur hinterlassen hat. Schmidt hat mir hier vor allem ein prinzipielles Bedenken .entgegengehalten, das der Beachtung wert und doch leicht aufzulösen ist. Er weist darauf hin, daß die Anschauung des Teufels als eines Fürsten der Unterwelt, der als Gegner Christi in Betracht kommen könne, sich weder im Judentum noch im Urchristentum finde, sondern erst viel später auftrete. Der Teufel sei z. B. noch in der Apokalypse des Johannes ein gefallener aus dem Himmel geworfener

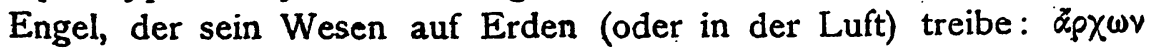

${ }^{1}$ Vgl. Zimmern, Ztschr. für Assyriologie XXXII S. 67 f. und XXIII S. 364 ff. Sicher gedeutet sind die Figuren des Enmes̉ara und seiner sieben Kinder allerdings noch nicht. 


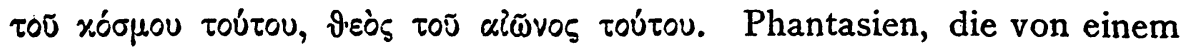
Kampf Christi mit dem Teufel in der Unterwelt redeten, seien erst einer späteren. Entwicklung zuzuweisen. Es soll der Einwand einmal als berechtigt zugestanden werden. Aber gab es denn in der jüdischen Phantasie keine anderen Mächte der Unterwelt, die als Gegner des Christos in Betracht kommen könnten? Um nun bei der Apokalypse zu bleiben, so erinnere ich meinen Kritiker an das Tier aus. dem Abgrunde, an den Abaddon-Apollyon, an die Personifikation des Todes und des Hades $(6,8)$. Ich erinnere ihn ferner an die ganz

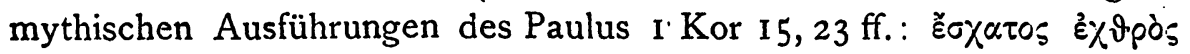

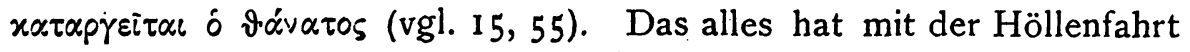
zunächst nichts zu tun, weist aber doch das Milieu auf, in welchem die Phantasien von einem Kampf Christi in der Unterwelt Wurzeln schlagen und wuchern konnten. Wenn der T e u fel nicht als Gegner Christi in Betracht kommt; so bietet sich zum Erșatz die genaue Figur des $\mathrm{H}$ ades oder des $\mathrm{Th}$ a $\mathrm{n}$ atos. Es ist doch charakteristisch, daß Plutarch (Theopomp); (de Iside et Os. 47) den persischen 'Aperuávios mit dem Hades gleichsetzt. Die Quellen, die direkt von der Hadesfahrt handeln, bieten eine erfreuliche Bestätigung. So finden wir bei dem christlichen Interpolator Testam. Lev. c. 4 die charak-

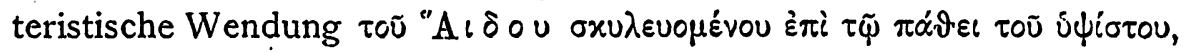
charakteristisch auch dann, wenn Schmidt mit Recht diese Andeutung nur auf Mt 27, 52 beziehen würde. Denn auch diese Stelle darf nicht so einfach, wie Schmidt dies tut, isoliert und aus dem Zusammenhang der Untersuchung über den Hadesmythos losgelöst werden. Bedeutsam ist auch die zweite in Betracht kommende Stelle Dan 5 :

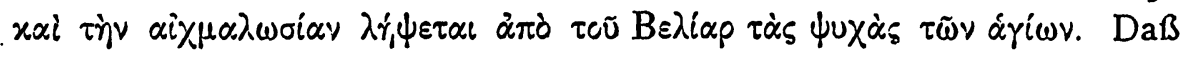

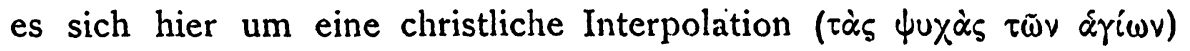
handelt, hat Schmidt richtig gesehen. Doch braucht die Interpolation nicht spät zu sein. Schmidt schließt das wieder aus dem allgemeinen Satz, daß die Vorstellung eines Herrschers der Unterwelt nicht nur in der jüdischen Apokalyptik, sondern auch in der christlichen Literatur ganz singulär sei. Aber bewiesen hat er doch höchstens nur dies, daß der Te ufel in jüdischer oder urchristlicher Auffassung nicht Herrscher der Unterwelt sei. Beliar ist aber nicht olne weiteres identisch mit dem Teufel ${ }^{1}$. Es ist die Vermutung hinreichend begründet, daß Beliar gerade nach älterer Anschauung ein Fürst der Unterwelt war; seine Identifikation mit dem aus dem Hades wiederkehrenden Nero weist noch darauf hin, daß diese Auffassung seines Wesens sich auch später noch erhalten hat. Ganz deutlich spricht

1 Vgl. meine Religion des Judentums S. $289,292 \mathrm{f} ., 384 \mathrm{f}$. 
hier dann weiter das Zeugnis der Himmelfahrt des Jesaja 9, 16: Und wenn er dem Engel des Todes die Beute genommen haben wird, wird er am dritten Tage auferstehn (vgl. 10, 10. I4; II, I9 > Und er stieg hinab zum Engel [der Unterwelt]). Wiederum heißt es in den

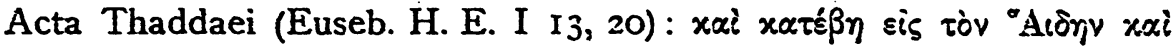

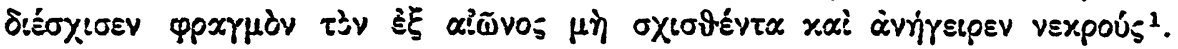
In diesen Zusammenhang rücken ferner die Thomasakten ein, in denen

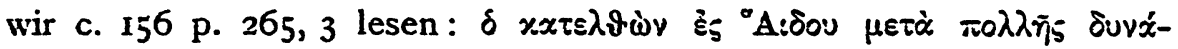

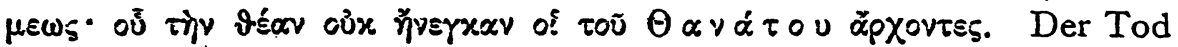
erscheint hier als der gewaltige Fürst der Unterwelt, von seinen Archonten umgeben ${ }^{2}$. Und endlich beginnt die 42 . Ode Salomos: -Die Unterwelt (sc. der Ha des) sah mich und ward elend, und der Tod spie mich aus und viele mit mir.« So begreift es sich dànn auch leicht, daß der Teufel, ursprünglich vielleicht nur >der Herrscher dieser Welte, mit dem $>$ Herrscher der Unterwelt ${ }^{3}{ }^{3}$ identifiziert wird oder neben diesem in der Unterwelt als Herrscher erscheint (Acta Thomae, Evangelium Nicodemi).

Auf Grund dieser Darlegungen halte ich denn auch an meiner Deutung von Apc I, I $\delta$ fest: Wenn hier der Menschengestaltige als der Besitzer der Schlüssel des Todes und des Hades erscheint, so liegt die mythische Vorstellung von Kampf und Sieg gegenüber den Mãchten der Unterwelt dem zugrunde. Und wenn Schmidt das eine moderne Eintragung in den Text nennt (S. 551), so kann ich den Einwand nicht gelten lassen, glaube vielmehr, daß mein Kritiker sich durch eine fast dogmatistisch verengende Betrachtung des jüdischen und urchristlichen Unterwelt-Bildes den Weg zu einer lebendigen Auffassung mit ihrer tatsächlichen Mannigfaltigkeit willkürlich versperrt hat. Auch an meiner Deutung von Mt 16 , 18 halte ich fest: Die $\pi \dot{\lambda} \lambda \alpha \iota{ }^{\circ A}$ A $\delta$ ou brauchen nicht gerade >das Reich des Satan. (Schmidt 55I) zu bedeuten; wenn man aber in dieser Wendung nur eine >Umschreibung bzw. einen pleonastischen Ausdruck für den Tod finden will, nur die Weissagung $>$ daß der leibliche Tod über Petrus nicht die. Oberhand gewinnen, d.h. daß dieser nicht sterben werde $\epsilon$, so vermag ich darin nur eine rationalistische Vergewaltigung eines mythischen Textes zu erblicken. Auch Mt 27,5 I möchte ich, wie schon gesagt, nach wie vor. in diesen Zusammenhang einreihen, doch

1 Vgl. auch die reviwopol "Aloou nach Hiob 38, 17 bei Hippolyt ed. Achelis I 2, $268 \mathrm{f}$.

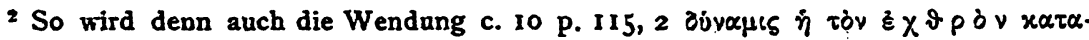

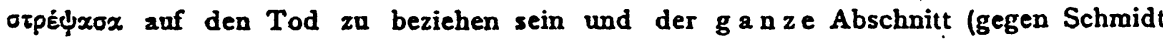
S. 555 f.) auf die Hadesfahrt. Darauf deutet auch die Wendung hin: $\dot{\eta} \varphi \omega v \dot{\eta} \dot{\eta}$ ¿xovo-

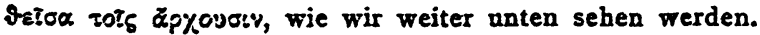

Vgl. Schmidt 559. 
will ich kein Gewicht ${ }^{*}$ auf diese Stelle legen. Ich komme nur noch einmal auf diese zurück, um noch einen Einwand allgemeiner Art, den mein Gegner bei dieser Gelegenheit gegen meine Gesamtauffassung erhebt, zu beleuchten. Er formuliert diesen: »Wäre wirklich die Idee von dem triumphierenden Sieger über die Mächte der Unterwelt in der urchristlichen Zeit lebendig gewesen, würde man schwerlich im Kerygma bekannt haben, daß Christus von Gott auferweckt sei (S. 552). „Daß der Messias aus eigener Kraft und eigenem Willen die Gewalt des Hades siegreich gebrochen hat, war nicht der nächstliegende Gedanke. « Ich vermag in diesen Sätzen nur wieder das Muster einer verkehrten dogmatistischen Betrachtung der christlichen Urzeit zu erblicken, die dem Reichtum und der Fülle geschichtlicher Erscheinungen nicht gerecht wird. Ich erinnere meinen Kritiker nur; um bei schon Gesagtem zu bleiben, an das Bild vom Menschengestaltigen in seiner selbsteigenen Herrlichkeit, das uns der Apokalyptiker in seinem ersten Kapitel zeichnet, an das gewaltige Wort sauf diesen Fels will i $\mathrm{ch}$ m e in e Kirche bauen «, ja selbst auf

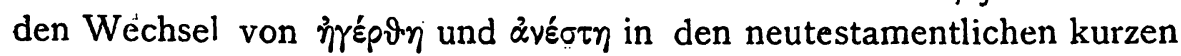
Bekenntnissen. $\mathrm{Zu}$ den Ausführungen, die mein Kritiker $\mathrm{zu}$ den späteren Zeugnissen der »volkstümlichen * Auffassung der Höllenfahrt macht, habe ich nur wenig hinzuzufügen. Er macht auch hier allerlei Abstriche. Bald findet er hier liturgische Sprache, welche sich gern in plastischen Bildern bewegt ${ }^{1}$, bald bemüht er sich den einzelnen Wendungen einen andern Sinn und Zusammenhang zu geben, bald will er in ihnen wesentlich poetische freie Ergüsse sehen. Besonders betont er des öfteren, daß in diesen Höllenfahrtschilderungen doch wenigstens von einem eigentlichen Kampf Christi in der Unterwelt keine Rede sein könne, insofern die Gegner sofort einen solchen furchtsam und verzagt aufgäben. Als wenn es mir auf derartige geringfügige und so leicht begreifliche Nuancen und Varianten ankäme! Ich will mich hier auf Einzelheiten nicht mehr einlassen, ich hebe lieber hervor, daß auch mein Kritiker mit der Möglichkeit rechnet, daß meine Auffassung hinsichtlich zweier sehr wichtiger Quellen der

${ }^{1}$ Das Recht der Deutung, die Schmidt S. $552 \mathrm{f}$. dem unter Melitos Namen umlaufenden Hymnus und dem betr. Passus der ägyptischen Kirchenordnung gibt, kann ich nicht zugestehen. Bei Melito ist es dürch die Reihenfolge der Sätze (surrexit e mortuis - pede decalcavit mortem et vinxit potentem) geradezu ausgeschlossen, an Besiegung von Tod und Teufel durch den Tod (im Sinn des Paulus) zu denken. Schließlich gibt doch auch Schmidt zu, daß hier Töne angeschlagen werden, die bci früheren Schriftstellem unbekannt waren. - Für mich handelt es sich hier nicht um Schriftsteller und Literatur, sondern um Liturgie, die Altertümliches und Volkstümliches am besten bewahrt. 
Thomasakten und der Oden Sąlomos zu Reçht bestehen könnte. Aber freilich die Thomasakten sind ihm 'ein Glied der syrischen Provinzialkirche, dessen Glaubensvorstellungen wir durchaus nicht mit dem gemeinchristlichen Volksglauben identifizieren dürfen (S. 560). -Der Sänger der Oden (Salomos) zeigt in seinen religiösen Ausdrucksformen und seinem theologischen Begriffsmaterial ganz individuelle Färbung und Haltung. ¿ Das ist dieselbe Methode, mit welcher der Verfasser das wichtigste neutestamentliche Zeugnis für die Hadesfahrt Christi, I Pt 3, 19; gleich im Anfang seiner Untersuchung mit einer Handbewegung beiseite schiebt. Ich fühle mich durch sie nicht veranlaßt, meine Auffassung aufzugeben, daß uns schon im Neuen - Testament Spuren einer volkstümlichen Auffassung von der Höllenfahrt Christi begegnen, die dann erst in späteren Quellen (altchristlicher Liturgie, Thomașakten, Oden Salomos) in ihrem vollen Umfang begegnen. Noch wichtiger und entscheidender aber ist mir der Nachweis, daß die in der Theologie des Urchristentums herrschend gewordene Anschauung von der Hadespredigt Christi ihre direkten Wurzeln im orientalischen Mythos hat. :

Endlich aber mag, wenn auch in aller Kürze, noch daraut hingewiesen werden, daß in diesem größeren Zusammenhang nun auch die beiden rätselhaften Aeußerungen des Apostels Paulus I Kor 2, 6-8 $\mathrm{Kol} 2,14 \mathrm{f}$. einzubeziehen sind, um ihr volles Licht zu erhalten und andrerseits Paulus als indirekten Zeugen eines alten Mythos von der Höllenfahrt zu erweisen.

Schmidt selbst weist in längeren Ausführungen seiner Untersuchung darauf hin, wie eine Reihe christlicher Gnostiker ebenfalls die Bekanntschaft mit der Hadesfahrt Christi voraussetze, nur daß sie den Schauplatz der Begebenheit nicht in die Unterwelt verlegen, sondern in die Himmelssphären, welche Christus bei seinem Aufstieg (resp. Herabstieg) zur Erde berührt. Man kann dies auch so formulieren, daß für eine weit verbreitete gnostische Anschauung sich das Erscheinen Christi auf Erden als Hadesfahrt darstellt, deren verschiedene Etappen sich als Durçhgang durch die Himmelssphären darstellen ${ }^{1}$. Schmidt sieht hier eine bewußte und sekundäre Umdeutung, welche gnostische Schulen an der kirchlichen Idee vorgenommen haben, und erblickt hier, nebenbei gesagt, eine feste Grenz-

1 Seinem ganzen Umfange nach könnte dies Problem erst unter Heranziehung der von Reitzenstein neu erschlossenen mandäischen und manichäischen Quellen behandelt werden. (Sitzungsber. d. Heidelb. Akad. 1919 S. 25 ff.) Das aber würde eine breitere Untersuchung erfordern, die ich hier nicht liefern kann. Ich begnüge mich, auf diese wichtigen Texte zum Mythos von der Hadesfahrt hinzuweisen. 
scheide zwischen Gnosis und Vulgärkatholizismus. Ich möchte mich hier vorsichtiger äußern und sehe in dieser Verlegung des Hades und seiner Schrecken in überirdische Räume einen allgemeinen Vorgang, der sich bereits in vorchristlicher Zeit vollzogen haben könnte. Die Umwandlung, welche vor allem die griechische Stoa den volkstümlichen Hadesvorstellungen nach dieser Richtung hin hat zuteil werden lassen, sind bekannt. Sie liegen in den eschatologischen Phantasien Plutarchs in besonders greifbarer Deutlichkeit vor. Ich 'weise im Vorbeigehen darauf hin, daß die gefallenen Engel, die nach dem äthiopischen Henochbuch in der Unterwelt gestraft werden, im slawischen Henochbuch bereits im zweiten Himmel erscheinen und ihre Straforte dort haben (c. 7 u. I8) ${ }^{1}$. So halte ich es zunächst für möglich, daß der vorchristliche Mythos von der Hadesfahrt bereits in den Abstieg eines Erlöserheros durch die verschiedenen Himmelsetagen umgedeutet werden konnte.

Den Beweis liefern jene obengenannten Stellen der paulinischen Briefe. Sie werden dann sofort deutlich, wenn wir annehmen dürfen, daß hier der Mythos von der Hadesfahrt eines Erlösersheros und seinem Kampf mit dẹn dämonischen Mächten auf den Abstieg des Christos zu Ende, seinen Todeskampf am Kreuz und den durch ihn errungenen Sieg umgedeutet wurde. Ob Paulus die Umdeutung selbst vollzogen hat, oder ob ihm bereits ein Muster vorlag, mag dahingestellt bleiben. Jedenfalls erscheint Paulus hier als ein Vorläufer der christlich gnostischen Spekulationen, nur daß er ganz anders wie sie, die durch ihren Doketismus daran verhindert waren, den Tod Christi in diesen Zusammenhang einstellen, in ihm den Höhepunkt des ganzen Dramas erkennen konnte.

Ich lege zum Beweise besonderes Gewicht auf einen Zug. I Kor 2, 6-8 wird vor allem betont, daß die Archonten den Herrn der Herrlichkeit, den sie ans Kreuz schlugen, nicht gekannt hätten. Nun ist bekanntlich das ein stereotyper Zug in allen gnostischen Phantasien, daß - der Erlöser den Mächten der einzelnen Himmelssphären bei seinem Abstieg unbekannt bleibt, indem er sich jeweilig in ihre Gestalten verwandelt. Selbst in der Himmelfahrt des Jesaja, die in ihrer gegenwärtigen Form allerdings bereits mehr vulgärkatholisch sein mag, ist die Phantasie in breiter Ausführlichkeit vorgetragen. In den Höllenfahrten des mandäischen Heros Hibil-Ziwa spielt er eine besondere Rolle. Er ist auch im allgemeinen Rahmen des Mythos durchaus verständlich und motiviert. Der Erlöserheros steigt unbe-

1 Dem entspricht, daß das Paradies und der Strafort der ungerechten Seelen tiberhaupt im dritten Himmel erscheinen (c. 8 ff.). 
kannt und unerkannt in die tiefsten Tiefen herab, um dort endlich den entscheidenden Schlag gegen die höllischen Mächte und Gewalten zu tun. Hibil-Ziwa läßt sich unerkannt von dem Höllenfürsten verschlingen und zerschneidet von ihm verschlungen seine Eingeweide von innen heraus. Das klingt noch in den Oden Salomos (42) nach: Der Hades sah mich und ward elend, und der Tod sp.ie $\mathrm{mich}$ a u s und viele mit mir. Für Paulus tritt die Peripetie mit dem Tode Christi ein. Die Archonten haben Christus ans Kreuz gebracht, ihn in den Tod verschlungen. Aber im Tode ist er ihnen übermächtig geworden, hat er den Mächten und Gewalten das Rüstzeug abgenommen und über sie triumphiert 1 .

Ist diese Kombination richtig, so erscheint nunmehr bereits Paulus als ein Zeuge für den volkstümlichen Mythos von einem Erlöserheros, der mit den Mächten der Tiefe kämpft, scheinbar unterliegt und triumphierend siegt. Doch möchte ich zum Schluß betonen, daß meine Auseinandersetzung mit Schmidt von diesen letzten Kombinationen vollkommen unabhängig ist, und bitte, diese als ein donum supererogationis $\mathrm{zu}$ betrachten.

Zum Schluß seiner Ausführungen betont Schmidt, daß er gegen eine allseitige religionsgeschichtliche Behandlung von der Hadesfahrt des Themas nichts einzuwenden habe, diese sogar mit Freuden begrüßen würde. , Aber mit ebenso großem Nachdruck muß ich den Standpunkt vertreten, daß der Religionshistoriker erst dann mit Erfolg an die Arbeit herantreten kann, wenn das Material innerhalb der einzelnen Religionen umfassend erforscht ist. Dann wird ihm zum Bewußtsein kommen, daß neben den mannigfaltigen Parallelen auch die großen Differenzen Berücksichtigung verlangen; sonst verfallen wir in einen Dilettantismus, der die religionswissenschaftliche Methode nur diskreditieren könnte. Dazu erlaube ich mir einige Gegénbemerkungen. Ich kann nicht anerkennen, daß mein Kritiker das für unsere Frage in Betracht kommende Material wirklich erst in um-

1 In diesen Zusammenhang gehört auch jenes Rätselwort hinein,. das in unsern Quellen von der Hadesfahrt so oft erscheint, und das Clemens Stromata VI 43 und

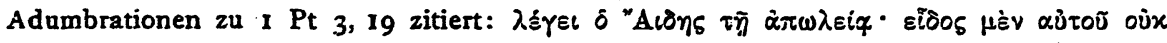

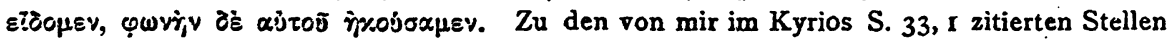
trage ich noch nach Hippolyt ed. Achelis $I^{2} 268$ und vor allem Acta Thomae c. 10

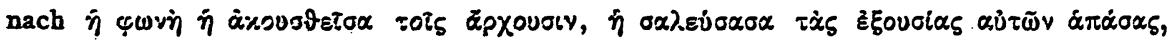
nebenbei (gegen Schmidt 555 f.) ein Beweis, daß die ganze Stelle auf die Hadesfahrt Christi zu beziehen ist, wobei die Frage gleichgültig bleibt, ob nicht eventuell auch bier die Archonten aus der Unterwelt in die himmlischen Sphären versetzt sind. (Vgl. auch mandäische und manichäische Parallelen bei Reitzenstein Sitz.Ber. d. Heidelb. Akademie 1919 S. 32, 1.)

Zeitschr. f. d. neutest. Wiss. 19. Jahrg. 1919/20. 
fassenderer Weise erforscht habe. Was er bietet, sind vielfach auf langen Seiten mühsame und gelehrte Einzeluntersuchungen, die zur Hauptfrage kaum noch Beziehungen haben. Seine Grundthese, die er nicht müde wird $z u$ verfechten, die weite Verbreitung der Lehre von der Hadespredigt Jesu für die Geister der alttestamentlichen Gerechten, war mir vor seiner Untersuchung zur Genüge bekannt und auch von mir betont. Berichtigungen in einigen wenigen Einzelheiten erkenne ich gern an. Unsere Hauptdifferenzen beruhen auf einer Beurteilung schon vorhandenen und bekannten Stoffes, und ich vermag mich hier nicht durch eine Arbeitsmethode umstimmen zu lassen, die beinahe dogmatisch die Linien der Betrachtung zieht, in diese allen Stoff hineinzwängt, entgegenstehende Beobachtungen fortwährend als Singularitäten mit gelassener Handbewegung beiseite schiebt und sich den eigentlichen Problemen andauernd verschließt. $\rightarrow$ Ich vermag auch die These nicht anzuerkennen, daß $z$ u $\mathrm{n} \ddot{\mathrm{ch}} \mathrm{st}$ das Material für die einzelne Religion umfassend erforscht sein müsse, ehe man zur religionsgeschichtlichen Betrachtung übergehe. An der umfassenden Erfassung des Materials liegt es mir ebensogut, wie meinem Kritiker. Ich bin aber der Meinung, daß erst der religionsgeschichtliche Vergleich auf diesem und auf anderm Gebiete eine umfassende und eindringende Forschung ermöglicht, bei der wirkliche Erkenntnis herausspringt und nicht bloße gelehrte Registratur-Arbeit und Vielwisserei, die keinen $Z$ weck hat. $\mathrm{Daß}$ man bei dem religionsgeschichtlichen Vergleich nicht bloß auf die Parallelen sondern auf die großen Diffe. renzen seinen Blick zu richten habe, ist für mich eine Binsenwahrheit. Mein Kritiker möge doch was ich in dieser Hinsicht über die Lehre von der Hadesfahrt Kyrios S. 39 gesagt habe, noch einmal lesen. Die Originälität christlichen Geistès strahlt nur in hellerem Licht, je genauer uns das Material bekannt wird, mit dem er arbeitete. - Die Warnung >vor einem Dilettantismus, der die religionswissenschaftliche Methode nur diskreditieren könnte «, lehne ich als eine Grenzüberschreitung gedeihlicher und friedlicher wissenschaftlicher Auseinandersetzung ruhig und bestimmt $a b$. 